\title{
Supercontinuum generation in three-fold symmetry microstructured fibers in visible and infrared spectral regions
}

\author{
Zbyszek Holdynski, ${ }^{* 1}$ Marek Napierala, ${ }^{1}$ Michalina Jozwik, ${ }^{1}$ Lukasz Szostkiewicz, ${ }^{1}$ Pawel Mergo ${ }^{2}$, \\ and Tomasz Nasilowski ${ }^{1}$ \\ ${ }^{1}$ InPhoTech Ltd., Slominskiego 17/31, 00-195 Warsaw, Poland, \\ ${ }^{2}$ Laboratory of Optical Fiber Technology, Maria Curie-Skłodowska University, Pl. Sklodowskiej 3, 20-031 Lublin, \\ Poland
}

Received March 10, 2016; accepted May 16, 2016; published June 30, 2016

\begin{abstract}
We present a series of threefold symmetric silica microstructured fibers for nonlinear application. The fibers are designed to have the zero dispersion wavelength far from the pump wavelength In our fibers the domination of nonlinear effects is precisely controlled by selectively increased three air holes in the vicinity of the core. Our fibers allow for broadband single mode spectrum generation and achievement of flat spectra even after the changes of fiber dispersion caused by fabrication inaccuracies.
\end{abstract}

For nonlinear applications (like broadband sources design), the zero dispersion wavelength (ZDW) is a fundamental parameter to control pulse propagation in optical fibers. The position of ZDW defines the normal and anomalous dispersion side and allows for different nonlinear effects domination by appropriate mode or phase matching condition application [1,2]. In terms of a sub-nanosecond pump (used in this letter) the nonlinear effects can be grouped in: Stimulated Raman Scattering (SRS), four wave mixing (FWM) and soliton dynamic in the anomalous dispersion side combined with dispersive waves generation in the visible wavelength range [1-3, 4]. Highly nonlinear suspended core fibers (SCF) have large advantages coming from the possibility of zero dispersion wavelength shift in an infrared and visible spectrum by special fibre design $[5,6]$. Decreasing the core size to the nanometer scale gives also efficient nonlinear effect generation [6]. The possibility to control dispersion shape and ZDW position in a wide spectral range and high nonlinearity level determines high application potential of SCFs. Unfortunately, SCFs are multimode which is unwanted in potential applications. In addition, a very small core size of SCFs enforces the fabrication of such fiber with very high precision to keep proper dispersion properties [8, 9-11, 12]. Therefore, it is difficult to predict dispersion characteristics in actual fibers due to fabrication inaccuracies.

In the paper, we focused on optimizing nonlinear fibers for stable spectrum generation even after large geometry changes. Stable spectrum generation can be obtained by pure domination of one nonlinear effect in the initial stage

\footnotetext{
*E-mail: zholdynski@inphotech.pl
}

of spectrum broadening. The popular condition of pumping close to ZDW gives a wide spectrum but fiber geometry changes can shift dispersion and significantly change the generated spectrum [13, 14]. We designed a series of fibers to obtain the condition of pumping far from ZDW in normal and anomalous dispersion regimes. We propose such a pumping condition as a method which gives stable spectra. In our investigations we present the possibility to merge advantages coming from both: single mode microstructure and high nonlinearity in one fibre. Special design of a hybrid microstructure with enlarged three air holes enables to control the ZDW position in a wide spectral range from the visible to infrared region. We show how the dynamics of nonlinear effect changes can be obtained by dispersion characteristic manipulation. In the paper we present three series of microstructured fibers. The first series consists of three pure silica photonic crystal fibers (fibers $A, B, C$ ) with three enlarged air holes around the core, see Fig. 1. The second series of microstructured fibers consists of fibers $D, E$ and $F$, in which ZDW is shifted to shorter wavelengths by increasing the air filing factor. On the other hand, the third series of fibers $(G, H$, and $I)$ has ZDW shifted to a longer wavelength side to obtain normal dispersion at $1064 \mathrm{~nm}$ pumping wavelength. All fibers were pumped by a sub-nanosecond light source at a wavelength of $1064 \mathrm{~nm}$ (pulse width $0.75 \mathrm{~ns}$; energy per pulse $7.6 \mu \mathrm{J}$; repetition rate $19.75 \mathrm{kHz}$; average power $50 \mathrm{~mW}$ ). Designed fibers geometry parameters were shown in Tab.1.

For anomalous dispersion regime pumping, initially the spectrum broadening starts from a cascade of the FWM process. In fibers $A, B, C$ extremely a fast and asymmetric broadening mechanism is observed since $\mathrm{ZDW}$ is far from the pump position. Pumping far from ZDW needs more power than pumping close to ZDW and it shifts the phase matching condition between dispersive waves and solitons to the shortest wavelength. In our case, pumping fibers $A, B$ and $C$ offers the possibility of matching infrared solitons and dispersive waves in the visible wavelength range, as presented in Fig. 2. Optimization of dispersive waves trapping by solitons needs a reduction of fiber loss in the infrared range. In fibers $A, B$, and $C$ the 
effect of fiber loss influence on visible part generation is clearly seen (see fiber A, B, C loss in Fig. 2.).

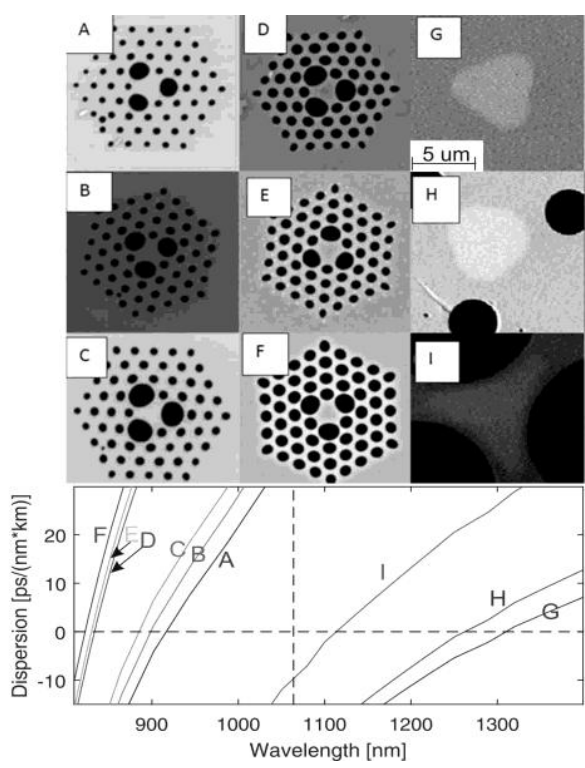

Fig. 1. Geometry of fibers and their fundamental mode dispersion. Dashed line represents pump wavelength position and zero dispersion level. Zero dispersion wavelength positions for fibers are respectively: $Z_{\mathrm{ZDW}}=1311 \mathrm{~nm} ; \mathrm{ZDW}_{\mathrm{H}}=1265 \mathrm{~nm} ; \mathrm{ZDW}_{\mathrm{I}}=1112 \mathrm{~nm} ; \mathrm{ZDW}_{\mathrm{A}}=916 \mathrm{~nm}$;

$Z_{\mathrm{ZDW}}=898 \mathrm{~nm} ; \mathrm{ZDW}_{\mathrm{C}}=885 \mathrm{~nm} ; \mathrm{ZDW}_{\mathrm{D}}=833 \mathrm{~nm} ; \mathrm{ZDW}_{\mathrm{E}}=829 \mathrm{~nm} ;$ $\mathrm{ZDW}_{\mathrm{F}}=823 \mathrm{~nm}$.

Table 1. Geometrical parameters of designed fibers

\begin{tabular}{|c|c|c|c|c|}
\hline Lp: & $\begin{array}{l}\text { Core } \\
\text { diameter } \\
{[\mu \mathrm{m}]}\end{array}$ & $\begin{array}{l}\text { Large air } \\
\text { holes } \\
\text { diameter }[\mu \mathrm{m}]\end{array}$ & $\begin{array}{l}\text { Lattice } \\
\text { constant[ } \\
\mu \mathrm{m}]\end{array}$ & $\begin{array}{l}\text { Small air } \\
\text { holes } \\
\text { diameter }[\mu \mathrm{m}]\end{array}$ \\
\hline$A$ & 3.0 & 2.4 & 2.3 & 0.8 \\
\hline$B$ & 2.8 & 2.8 & 2.6 & 1.1 \\
\hline$C$ & 2.7 & 3.1 & 2.7 & 1.0 \\
\hline$D$ & 2.2 & 2.2 & 1.8 & 1.0 \\
\hline$E$ & 2.1 & 1.8 & 1.6 & 1.0 \\
\hline$F$ & 2.0 & 1.8 & 1.6 & 1.2 \\
\hline$G$ & 8.0 & - & - & \\
\hline$H$ & 8.0 & 6.2 & 9.4 & \\
\hline$I$ & 5.5 & 18.2 & 9.9 & \\
\hline
\end{tabular}

The transmission characteristic in fibers $A, B, C$ was analyzed for 1-meter length of each fiber from Fig. 2. Extremely low transmission in fiber $\mathrm{A}$ in the infrared region exerts a major influence on the spectrum broadening limit, Fig. 3.

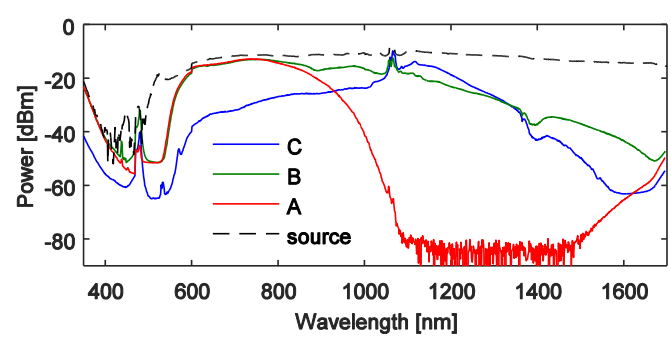

Fig. 2. Transmission characteristic in designed fibers $A, B, C$ (dashed lines presents source reference).
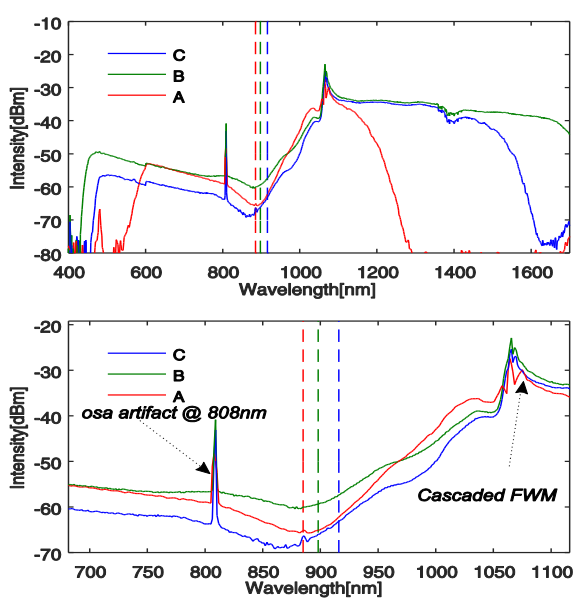

Fig. 3. Nonlinear effect generation in designed fibres $A, B, C$ with the domination of a cascaded FWM nonlinear process (dashed lines presents ZDWs).

The large distance between $\mathrm{ZDW}$ and the pump wavelength gives deep at a wavelength range of 850900nm (see Fig. 3). Fiber $A$ with small air holes' presents narrow bandwidth because of a large loss at infrared (reduction of infrared soliton shift). Fibers $B$ and $C$ with larger holes give a better view of the fundamental mode and have a smaller loss in the infrared region. Thus, solitons can reach the long wavelength side, in which the phase matching condition exists with dispersive waves in the visible wavelength range, and broadband spectra are obtained. In fibers $D, E, F$ the larger distance between ZDW and the pump position allows only the IR part of the spectra to be created (see Fig. 4).
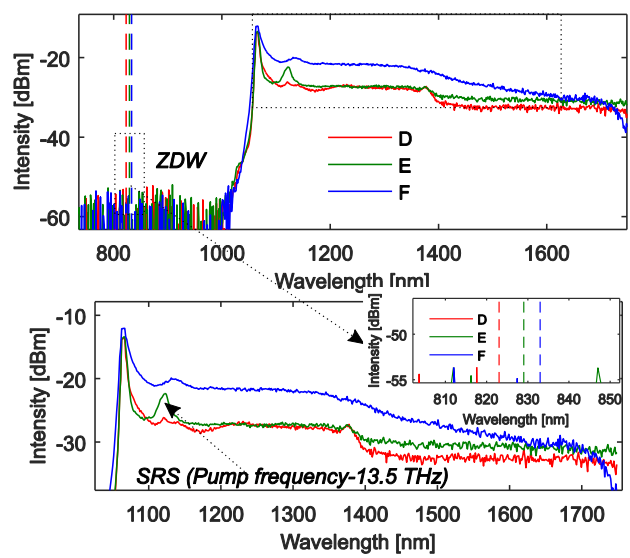

Fig. 4. Nonlinear effect generation in designed fibres $D, E, F$ (dashed lines presents ZDW). Registered spectral noise above $1400 \mathrm{~nm}$ wavelength can be the effect of pump source stability and decreased efficiency of supercontinuum generation. SRS peak frequency position at $13.5 \mathrm{THz}$ from pump wavelength.

In Figure 4 the spectra generated by pumping extremely far from $\mathrm{ZDW}$ are shown. In such a pumping condition the spectrum is initially broadened by the cascaded FWM. However, it produces an extremely narrow parametric gain (not shown in the article but verified experimentally 
by pump power changes). In fibers $D, E$ and $F$ the order of solitons and their broadening process are also reduced. The limitation in the soliton broadening process does not allow reaching a normal dispersion region for efficient dispersive wave emission. Spectra generated on the anomalous dispersion side are created by SRS mechanisms (registered, which dominate at longer wavelengths and spectrally flat spectra are generated in the whole telecom spectral range $( \pm 3 \mathrm{dBm}$ power difference in a range of $1200 \div 1700 \mathrm{~nm}$ ). Fibers $D, E, F$ have a different $\mathrm{ZDW}$ position but their difference plays a minor role in the generated spectrum shape.

The last series of microstructured fibers $(G, H, I)$ enables to achieve the pumping condition far from $\mathrm{ZDW}$ but the pump is localized in a normal dispersion region. Fiber G without air holes has ZDW located at $1311 \mathrm{~nm}$ and extremely deep normal dispersion regime pumping is obtained. In fiber $H$ the air holes enable the shift of ZDW to $1265 \mathrm{~nm}$ without changing nonlinear properties as compared to fibre $G$. The air holes' diameter of $6.2 \mu \mathrm{m}$ enables to create a narrow bandwidth initiated by the SRS process (frequency representation of generated SRS peaks are shifted $13.5 \mathrm{THz}$ from the pump position). The spectrum generated in fiber $H$ slightly crosses the ZDW position and starts to produce soliton, as shown in Fig.5. Geometry $I$ consists of three large air holes which shift ZDW to $1112 \mathrm{~nm}$. Normal dispersion regime pumping enables to obtain SRS spectra. In fiber $I$ where the ZDW position is close enough to the pump wavelength, the parametric FWM process is also observed. The domination of SRS and FWM effects (registered by pump power changes) in fiber I creates a broadband spectrum (see Fig. 5).
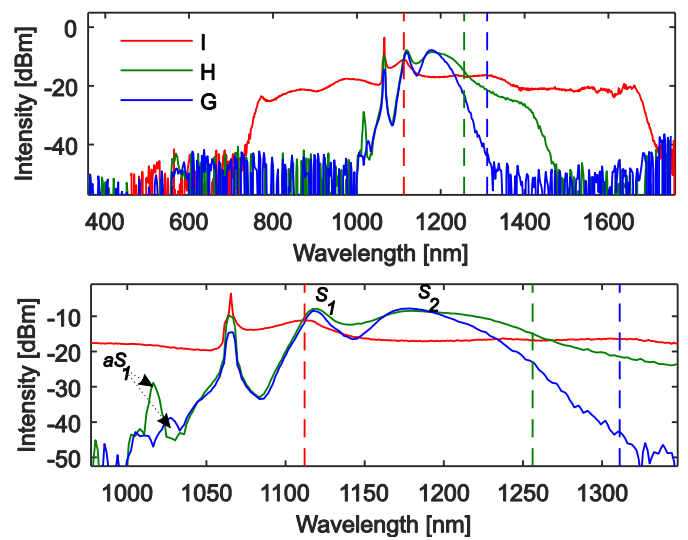

Fig. 5. Nonlinear effect generation in designed fibres $G, H$, $I$ with domination of SRS nonlinear process (where $\mathrm{aS}_{1}$ : anti-Stokes peak, $\mathrm{S}_{1}, \mathrm{~S}_{2}$ : Stokes peaks; dashed lines presents ZDW)

The diameter of air holes in fiber $I$ was increased to $18 \mu \mathrm{m}$ to obtain the ZDW shift closer to $1064 \mathrm{~nm}$, but still allowing normal dispersion regime pumping. The use of large diameter air holes makes the fiber less susceptible to fabrication inaccuracies. In fiber $\mathrm{G}, \mathrm{H}$, and $\mathrm{I}$, the fundamental mode is dominant but higher order modes also can be excited (opposite to previous geometries). Future investigations need to be applied to analyze single mode propagation in such geometries.

In conclusion, we have presented microstructured fibers with three enlarged air holes around the core, which offers the possibility of shifting ZDW near the visible wavelengths. Threefold symmetry fibers offer the possibility to precisely control nonlinear effects domination, which plays a major role in spectrum broadening. We have shown that nonlinear effect domination can be controlled in a simple way when fibers are pumped far from ZDW (e.g. in fibers, $D, E, F$ and $G$, $H$ ). In addition, deep anomalous dispersion regime pumping and highly nonlinear properties of fibers $D, E$ and $F$ produce broadband and flat spectra in the whole telecom spectral range. The narrow spectrum generated by SRS effects in fiber with large structural parameters is less sensitive to geometrical changes (see the difference between spectra $G$ and $H$ ). Our results can be used to design a flat $( \pm 3 \mathrm{dBm}$ fibers $D$ and $E)$ spectrum or narrowband high power sources (fibers $G, H$ ) to e applied in precise measurements and quality control in the telecom spectral range.

The work described in this paper was partially supported by the Polish National Science Centre within the project 2013/09/N/ST7/04429

\section{References}

[1] S.T. Sørensen, C. Larsen, C. Jakobsen, C. L. Thomsen, O. Bang, Opt. Lett. 39, 1097 (2014).

[2] V. Couderc, P. Leproux, V. Tombelaine, L. Grossard, A. Barthélémy, Opt. Expr. 13, 8584 (2005).

[3] H.E. Heidepriem, S.C. W. Smith, T.M. Monro, Opt. Expr. 17, 2646 (2009).

[4] U. Møller, Y. Yu, I. Kubat, C. R. Petersen, X. Gai, L. Brilland, D. M'echin, C. Caillaud, J. Troles, B. Davies, O. Bang, Opt. Expr. 17, 3282 (2015).

[5] I. Shavrin, S. Novotny, H. Ludvigsen, Opt. Expr. 21, 32141 (2013).

[6] M. Klimczak, G. Stepniewski, H. Bookey, A. Szolno, R. Stepien, D. Pysz, A. Kar, A. Waddie, M.R. Taghizadeh, R. Buczynski, Opt. Lett. 39, 2241 (2014)

[7] X. Zhang, X. Zhu, X. Chen, H. Li, J. Peng, N. Dai, J. Li, Opt. Expr. 20, 19799 (2012)

[8] C. Lesvigne, V. Couderc, A. Tonello, P. Leproux, A. Barthélémy, S. Lacroix, F. Druon, P. Blandin, M. Hanna, P. Georges, Opt. Lett. 32 2173 (2007).

[9] S. T. Sørensen, U. Møller, C. Larsen, P.M. Moselund, C. Jakobsen, J. Johansen, T.V. Andersen, C.L. Thomsen, O. Bang, Opt. Expr. 20 10635 (2012).

[10] A. Kudlinski, M. Lelek, B. Barviau, L. Audry, A. Mussot, Opt. Expr. 18, $16640(2010)$

[11] A. Kudlinski, G. Bouwmans, O. Vanvincq, Y. Quiquempois, A. Le Rouge, L. Bigot, G. Mélin, A. Mussot, Opt. Lett. 34, 3631 (2009).

[12] A. Kudlinski, V. Pureur, G. Bouwmans, A. Mussot, Opt. Lett. 33, 2488 (2008)

[13] Z. Holdynski, M. Napierala, P. Mergo, T. Nasilowski, J. Lightwave Technol. 33, 2106 (2015)

[14] U. Møller, S.T. Sørensen, C. Larsen, P.M. Moselund, C. Jakobsen J. Johansen, C.L. Thomsen, O. Bang, Opt. Fiber Technol. 18, 3104 (2012) 